\title{
Thermal expansion effect on thickness-shear vibrations in a piezoelectric quartz filter with dot-ring electrodes
}

\author{
Xiangyang $\mathrm{Li}^{1}$, Jing $\mathrm{Liu}^{1,2 *}$ \\ ${ }^{1}$ School of Mechanical and Electrical Engineering, Ningbo Dahongying University, Ningbo 315175, China \\ ${ }^{2}$ Piezoelectric Device Laboratory, School of Mechanical Engineering and Mechanics, Ningbo University, Ningbo 315211, \\ China
}

Corresponding Author Email: liujing@nbdhyu.edu.cn

https://doi.org/10.18280/ijht.360223

Received: 27 September 2017

Accepted: 3 February 2018

\section{Keywords:}

quartz crystals, thickness-shear vibration, filter, thermal expansion effect

\begin{abstract}
This paper aims to disclose the thermal expansion effect on thickness-shear vibrations of quartz filters. To this end, a brand-new "dot-ring" electrode structure was proposed for quartz filter operating in thickness-shear modes (TSMs). Then, a reasonable solution to the government equations of thermal expansion was derived, and the thermal expansion effect on the operation modes and vibration frequencies of the filter were examined in details. Through the rational design of electrode size and quartz plate thickness, a frequency interval was determined with two trapped modes. Under the working temperature between $-55^{\circ} \mathrm{C}$ and $85^{\circ} \mathrm{C}$, the electrode sizes changed with the thermal expansion; the resonance frequency declined with the increase of temperature. However, the frequency difference considering the thermal expansion effect is extremely small, indicating that the quartz crystal filter has good thermal stability. The research findings shed new light on the design of quartz filters.
\end{abstract}

\section{INTRODUCTION}

Piezoelectric crystals are the main raw materials of acoustic wave resonators, filters and sensors. For instance, AT-cut quartz crystals are often made into acoustic wave devices which operate in the thickness-shear mode (TSM) of a plate $[1,2]$. It is extremely difficult to simulate these materials with 3D theory of linear piezoelectricity, due to their high anisotropy and electromechanical coupling. A viable alternative is to develop approximate $2 \mathrm{D}$ plate equations, such as the single scalar differential equation for the fundamental and the overtone TSMs. This equation was derived for AT-cut quartz plates [3-6], and later applied to other anisotropic materials [7-9].

The traditional design of resonators contains square/rectangular dielectric and piezoelectric layers and square electrodes [9-11]. In crystal filters, however, two square electrodes are arranged above the piezoelectric crystals. The acoustic waves from the square electrodes propagate in all directions [12-13], leading to concentrated electric field, stress and temperature [14] in the electrode corners. In this case, the materials may deteriorate or even fail. Therefore, it is meaningful to consider the thermal expansion effect on thickness-shear vibrations. Nevertheless, there is little theoretical analysis on this effect of crystal filters, because the situation is too complicated to be explained clearly with $2 \mathrm{D}$ equations [15-20].

Considering the above, this paper proposes a brand-new "dot-ring" electrode structure for quartz filter. The corner-less design of the structure helps eliminate the concentration of electric field. Then, a theoretical analysis was performed to acquire the basic vibration features of the quartz filter with the proposed electrodes, and investigate the thermal expansion effect.

\section{THERMAL EXPANSION}

The research object is an AT-cut quartz plate filter. As shown in Figure 1, the plate is $2 h$ in thickness and $\rho$ in mass density. There is a thin, elliptical dot electrode in the central region and a ring electrode in the annular region atop the quartz plate. The two electrodes form the "dot-ring" structure. The mass density and thickness of both electrodes are denoted as $\rho^{\prime}$ and $2 h^{\prime}$, respectively. The semi-major axis of the elliptical electrode points to the $x_{1}$ direction, while the semi-minor axis of the elliptical electrode points to the $x_{3}$ direction. The two axes are respectively denoted as $a_{i} R_{i}$ and

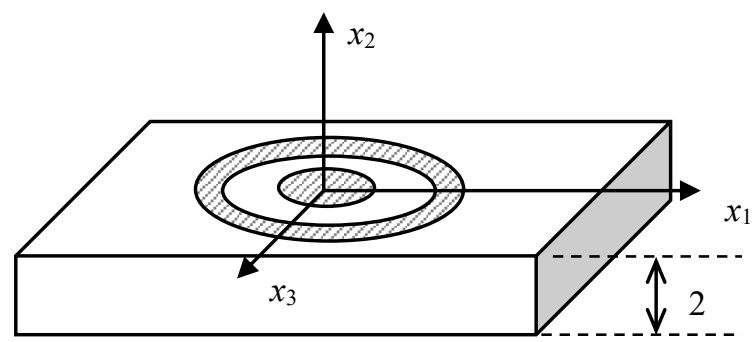

Figure 1. An FBAR filter with dot-ring driving electrodes

$R_{i}$, where $\mathrm{i}=0,1$ and 2 and the ratio $a_{i}$ will be determined by the later formula.

The thermal expansion coefficient can be expressed as [21]:

$\alpha=\Delta L /(L * \Delta T)$.

The electrode radius and the quartz plate thickness both change under thermal expansion. Considering the linear expansion coefficient of quartz crystal filter, the linear 
Table 1. The linear thermal expansion coefficients of the $x_{1}$ and $x_{2}$ axis

\begin{tabular}{cccccccccc}
\hline $\mathrm{T}\left({ }^{\circ} \mathrm{C}\right)$ & -250 & -200 & -100 & 0 & 100 & 200 & 300 & 400 & 500 \\
$\alpha_{1} \times 10^{-6}\left(/{ }^{\circ} \mathrm{C}\right)$ & 8.60 & 9.90 & 11.92 & 13.24 & 14.45 & 15.61 & 16.89 & 18.5 & 20.91 \\
$\alpha_{2} \times 10^{-6}\left(/{ }^{\circ} \mathrm{C}\right)$ & 4.10 & 5.50 & 6.08 & 7.10 & 7.79 & 8.75 & 9.60 & 10.65 & 12.22 \\
\hline
\end{tabular}

The linear expansion coefficient at any angle $\varphi$ relative to axis $x_{2}$ can be expressed as [21]:

$\alpha_{L}=\alpha_{2}+\left(\alpha_{1}-\alpha_{2}\right) \sin ^{2} \varphi$

At room temperature, $\alpha_{L}=\left(7.48+6.23 \sin ^{2} \varphi\right) \times 10^{-6}$.

\section{GOVERNING EQUATIONS}

For time-harmonic motions of a filter at resonance frequency $\omega$, all fields have the samei time-dependence with a common factor $\exp (i \omega t)$. Thus, the TSM displacement of order $n$ can be expressed as [3-6]:

$u_{1}^{n}\left(x_{1}, x_{2}, x_{3}, t\right)=\sum_{n=1,3,5 \ldots}^{\infty} u_{1}^{n}\left(x_{1}, x_{3}, t\right) \sin \left(\eta_{n} x_{2}\right)$

I there is no top driving electrode, the in-plane variation $u_{1}^{n}\left(x_{1}, x_{3}\right)$ in the TSM mode of order $n$ is governed by[3-6]:

$M_{n} \frac{\partial^{2} u_{1}^{n}}{\partial x_{1}^{2}}+c_{55} \frac{\partial^{2} u_{1}^{n}}{\partial x_{3}^{2}}-\frac{n^{2} \pi^{2} \bar{c}_{66}}{4 h^{2}} u_{1}^{n}=\rho \ddot{u}_{1}^{n}$

If there is a driving electrode, the in-plane variation $u_{1}^{n}\left(x_{1}, x_{3}\right)$ in the TSM mode of order $n$ is governed by:

$M_{n} \frac{\partial^{2} u_{1}^{n}}{\partial x_{1}^{2}}+c_{55} \frac{\partial^{2} u_{1}^{n}}{\partial x_{3}^{2}}-\frac{n^{2} \pi^{2} \hat{c}_{66}}{4 h^{2}} u_{1}^{n}=\rho \ddot{u}_{1}^{n}$

In $\mathrm{Eq}(4)$ and $\mathrm{Eq}(5), c_{55}$ is the elastic constant of quartz crystals. The $M_{\mathrm{n}}$ can be determined by:

$$
\begin{aligned}
M_{n}= & c_{11}+\left(c_{12}+c_{66}\right) \gamma \\
& +\frac{4\left(\gamma \bar{c}_{66}-c_{66}\right)\left(\gamma c_{22}+c_{12}\right)}{c_{22} n \pi \kappa} \cot \frac{\kappa n \pi}{2},
\end{aligned}
$$

where $c_{11}, c_{12}, c_{22}$ and $c_{66}$ are the elastic constants of quartz crystals. The coefficients $\bar{c}_{66}, \hat{c}_{66}, \gamma$ and $K$ can be obtained by:

$\bar{c}_{66}=c_{66}+\frac{e_{26}^{2}}{\varepsilon_{22}}, \kappa=\left(\frac{\bar{c}_{66}}{c_{22}}\right)^{1 / 2}, \gamma=\frac{c_{12}+c_{66}}{\bar{c}_{66}-c_{22}}$,

$\hat{c}_{66}=\bar{c}_{66}\left(1-\frac{8 \bar{k}_{26}^{2}}{n^{2} \pi^{2}}-2 R^{\prime}\right)$,

where $e_{26}$ and $\varepsilon_{22}$ are the piezoelectric constants and dielectric constants of quartz crystals, respectively. These constants can be determined by:

$$
\bar{k}_{26}^{2}=\frac{e_{26}^{2}}{\bar{c}_{66} \varepsilon_{22}}, R^{\prime}=\frac{2 \rho^{\prime} h^{\prime}}{\rho h} .
$$

In the $\left(x_{1}, x_{3}\right)$ plane, the elliptical electrode can be described as:

$\frac{x_{1}^{2}}{R_{i}^{2} M_{n} / c_{55}}+\frac{x_{3}^{2}}{R_{i}^{2}}=1, \quad i=0,1,2$.

The semi-major and semi-minor axes are $\sqrt{M_{n} / c_{55}} R_{i}$ and $R_{i}$, respectively. Then, a new coordinate system $\left(\lambda_{l}, \lambda_{3}\right)$ was introduced as:

$x_{1}=\lambda_{1} \sqrt{M_{n} / c_{55}}, \quad x_{3}=\lambda_{3}$.

The ellipses in $\mathrm{Eq}(10)$ can be converted into circle equations:

$\frac{\lambda_{1}^{2}}{R_{i}^{2}}+\frac{\lambda_{3}^{2}}{R_{i}^{2}}=1, \quad i=0,1,2$.

Then, the polar coordinates were introduced: letdependence $\theta$ of the fields be $\cos (m \theta)$ where $m=0,1,2 \ldots$ Thus, $\operatorname{Eq}(5)$ and $\mathrm{Eq}(4)$ can be rewritten as:

$\frac{\partial^{2} u_{1}^{n}(r)}{\partial r^{2}}+\frac{1}{r} \frac{\partial u_{1}^{n}(r)}{\partial r}-\frac{m^{2}}{r^{2}} u_{1}^{n}(r)+\rho c_{55}^{-1}\left(\omega^{2}-\hat{\omega}_{\infty}^{2}\right) u_{1}^{n}(r)=0$,

$\frac{\partial^{2} u_{1}^{n}(r)}{\partial r^{2}}+\frac{1}{r} \frac{\partial u_{1}^{n}(r)}{\partial r}-\frac{m^{2}}{r^{2}} u_{1}^{n}(r)-\rho c_{55}^{-1}\left(\bar{\omega}_{\infty}^{2}-\omega^{2}\right) u_{1}^{n}(r)=0$,

where $\hat{\omega}_{\infty}$ and $\bar{\omega}_{\infty}$ are the unbounded plate TSM resonant frequencies for plates with or without electrode, respectively. The two parameters obey $\bar{\omega}_{\infty}>\hat{\omega}_{\infty}$ because the resonant frequencies are dragged down by electrode inertia. The $\hat{\omega}_{\infty}$ and $\bar{\omega}_{\infty}$ can be described as:

$\hat{\omega}_{\infty}^{2}=\frac{n^{2} \pi^{2} \hat{c}_{66}}{4 \rho h^{2}}, \bar{\omega}_{\infty}^{2}=\frac{n^{2} \pi^{2} \bar{c}_{66}}{4 \rho h^{2}}$.

For "trapped modes", the vibration gradually attenuates away from an electrode edge, especially when $r$ approaches infinity. The boundary conditions are:

$u_{1}^{n}$ is finite, $r=0$,

$u_{1}^{n} \rightarrow 0, \quad r \rightarrow \infty$. 


\section{ANALYTICAL SOLUTION}

The following conditions are required to solve $\mathrm{Eq}(13)$ :

$\beta_{1}^{2}=\rho c_{55}^{-1}\left(\omega^{2}-\hat{\omega}_{\infty}^{2}\right)>0$,

$\beta_{2}^{2}=\rho c_{55}^{-1}\left(\bar{\omega}_{\infty}^{2}-\omega^{2}\right)>0$.

The above equation defines a frequency interval for the trapped modes of interest: $\hat{\omega}_{\infty}<\omega<\bar{\omega}_{\infty}$. Based on the separate variables in polar coordinates, we have:

$\xi_{1}=\beta_{1} r, \quad r<R_{0}$ and $R_{1}<r<R_{2}$,

$\xi_{2}=\beta_{2} r, \quad R_{0}<r<R_{1}$ and $R_{2}<r<\infty$.

Then, $\mathrm{Eq}(13)$ can be converted into:

$$
\begin{gathered}
\frac{\partial^{2} u_{1}^{n}\left(\xi_{1}\right)}{\partial \xi_{1}^{2}}+\frac{1}{\xi_{1}} \frac{\partial u_{1}^{n}\left(\xi_{1}\right)}{\partial \xi_{1}}+\left(1-\frac{m^{2}}{\xi_{1}^{2}}\right) u_{1}^{n}\left(\xi_{1}\right)=0, \\
0<r<R_{0} \text { and } R_{1}<r<R_{2}, \\
\frac{\partial^{2} u_{1}^{n}\left(\xi_{2}\right)}{\partial \xi_{2}^{2}}+\frac{1}{\xi_{2}} \frac{\partial u_{1}^{n}\left(\xi_{2}\right)}{\partial \xi_{2}}-\left(1+\frac{m^{2}}{\xi_{2}^{2}}\right) u_{1}^{n}\left(\xi_{2}\right)=0, \\
R_{0}<r<R_{1} \text { and } R_{2}<r<\infty .
\end{gathered}
$$

The two sub-equations of $\mathrm{Eq}(18)$ are the Bessel function and modified Bessel function of order $m$, respectively. Then, the general solution to $\mathrm{Eq}(18)$ satisfying $\mathrm{Eq}(15)$ can be written as:

$$
\begin{gathered}
u_{1}^{n}(r, \theta)=A_{1} J_{m}\left(\beta_{1} r\right) \cos (m \theta), \\
0<r<R_{0}, \\
u_{1}^{n}(r, \theta)=\left(A_{2} I_{m}\left(\beta_{2} r\right)+A_{3} K_{m}\left(\beta_{2} r\right)\right) \cos (m \theta), \\
R_{0}<r<R_{1}, \\
u_{1}^{n}(r, \theta)=\left(A_{4} J_{m}\left(\beta_{1} r\right)+A_{5} Y_{m}\left(\beta_{1} r\right)\right) \cos (m \theta), \\
R_{1}<r<R_{2},
\end{gathered}
$$

$u_{1}^{n}(r, \theta)=A_{6} K_{m}\left(\beta_{2} r\right) \cos (m \theta)$,

$$
R_{2}<r<\infty,
$$

where $A_{1} \sim A_{6}$ are unknown constants; $J_{m}$ and $Y_{m}$ are the mthorder Bessel functions of the first kind and second kind; $I_{m}$ and $K_{m}$ are the mth-order modified Bessel functions of the first kind and second kind. The solution to Eq(19) must satisfy the following continuous conditions:

$$
\begin{aligned}
& u_{1}^{n}\left(R_{0}^{-}\right)=u_{1}^{n}\left(R_{0}^{+}\right),\left.\quad \frac{d u_{1}^{n}}{d r}\right|_{R_{0}^{-}}=\left.\frac{d u_{1}^{n}}{d r}\right|_{R_{0}^{+}}, \\
& u_{1}^{n}\left(R_{1}^{-}\right)=u_{1}^{n}\left(R_{1}^{+}\right),\left.\frac{d u_{1}^{n}}{d r}\right|_{R_{1}^{-}}=\left.\frac{d u_{1}^{n}}{d r}\right|_{R_{1}^{+}}, \\
& u_{1}^{n}\left(R_{2}^{-}\right)=u_{1}^{n}\left(R_{2}^{+}\right),\left.\quad \frac{d u_{1}^{n}}{d r}\right|_{R_{2}^{-}}=\left.\frac{d u_{1}^{n}}{d r}\right|_{R_{2}^{+}} .
\end{aligned}
$$

Substituting $\mathrm{Eq}(19)$ into $\mathrm{Eq}(20)$, we have six linear homogeneous equations for $A_{1} \sim A_{6}$. For nontrivial solutions, the determinant of the coefficient matrix should be eliminated, producing the frequency equation. The modes can be determined by the corresponding nontrivial solutions of $A_{1} \sim A_{6}$. The numerical solutions are obtained with Matlab by selecting appropriate parameters.

\section{NUMERICAL SIMULATION AND RESULTS ANALYSIS}

The numerical example is a filter operating at the fundamental TSMs. For the remainder of this paper, the

values of $n$ and $m$ are fixed as $n=1$ and $m=0$. The other parameters are as follows: electrode-plate mass ratio $R^{\prime}=0.1$, plate thickness $2 h=200 \mu \mathrm{m}$, electrodes radii $R_{0}=620 \mu \mathrm{m}$, $R_{1}=720 \mu \mathrm{m}$ and $R_{2}=1,500 \mu \mathrm{m}$. The trapped modes are the interest modes and found in the narrow frequency interval determined by $\mathrm{Eq}(14)$. By this equation, it is determined that $\hat{\omega}_{\infty}=4.6492 \times 10^{7} \mathrm{rad} / \mathrm{s}$ and $\bar{\omega}_{\infty}=5.2185 \times 10^{7} \mathrm{rad} / \mathrm{s}$. The two trapped modes of interest were found in the frequency interval $\left(\hat{\omega}_{\infty}, \bar{\omega}_{\infty}\right) \quad: \quad \omega_{1}=4.9361 \times 10^{7} \mathrm{rad} / \mathrm{s} \quad$ and $\omega_{1}=4.9917 \times 10^{7} \mathrm{rad} / \mathrm{s}$, in this case.

Table 2. Numerical value of electrode radius and plate thickness at different temperatures

\begin{tabular}{ccccc}
\hline$T\left({ }^{\circ} \mathrm{C}\right)$ & $R_{0}(\mu \mathrm{m})$ & $R_{l}(\mu \mathrm{m})$ & $R_{2}(\mu \mathrm{m})$ & $h(\mu \mathrm{m})$ \\
\hline-55 & 619.35 & 719.25 & $1,498.44$ & 99.95 \\
-35 & 619.51 & 719.43 & $1,498.82$ & 99.96 \\
-15 & 619.67 & 719.62 & $1,499.20$ & 99.97 \\
5 & 619.83 & 719.81 & $1,499.60$ & 99.99 \\
25 & 620 & 720 & 1,500 & 100 \\
45 & 620.17 & 720.20 & $1,500.41$ & 100.01 \\
65 & 620.34 & 720.40 & $1,500.83$ & 100.03 \\
85 & 620.52 & 720.60 & $1,501.25$ & 100.04 \\
\hline
\end{tabular}

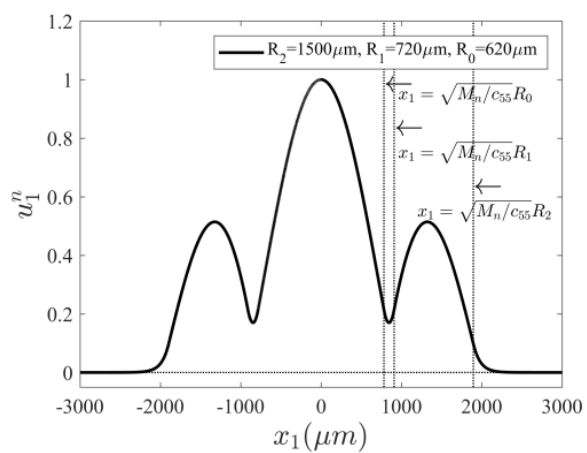

(a)

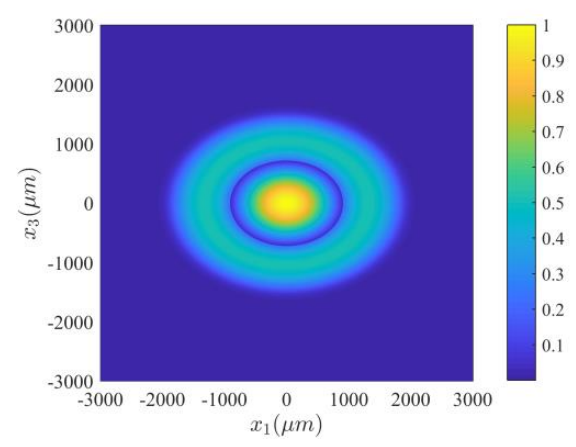



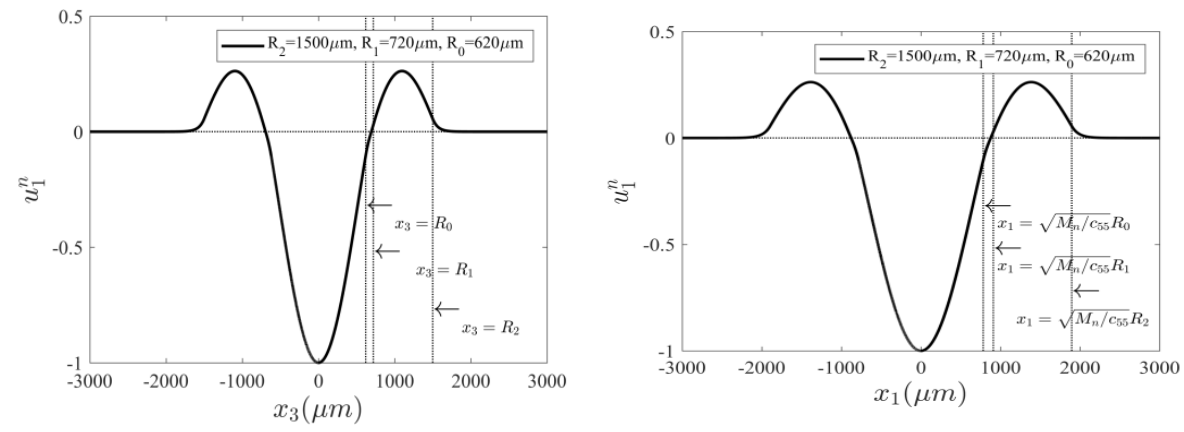

(b)

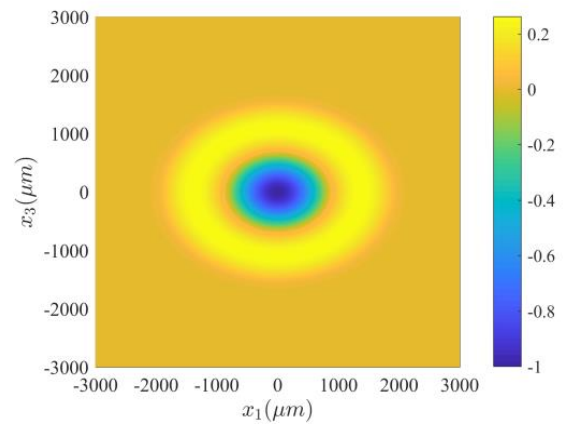

Figure 2. Two trapped thickness-extensional modes in the frequency interval

The two trapped modes bear high resemblance with each other, with a weak dependence on the in-plane mode variation. Figure 2 illustrates the cross-section of the two modes along $x_{1}=0$ and $x_{3}=0$. The mode in Figure 2(a) has no nodal line (point) except at the edges, but there exists a gap between the ring and dot electrodes. The mode vibrated under the dot electrode, attenuated between the ring and dot electrodes, and vibrated again under the ring electrode. The thickness-shear strain was always positive thanks to the thickness-shear displacement with $n=1$. The mode in Figure 2(b) has one nodal line between the ring and dot electrodes. The two sides of that nodal line vibrated in opposite directions. However, the charge of the electrode system is not cancelled out.

For better view, the contours of the two modes are also presented in Figure 2. It can be seen that the electrodes are oval in shape, and the semi-major axis $\left(\sqrt{M_{1} / c_{55}} R_{i}\right)$ and semiminor axis $\left(R_{i}\right)$ are along axes $x_{1}$ and $x_{3}$, respectively. The ratio between them was 1.47 , which is very close to the optimal electrode shape in References [22-24].

In the numerical simulation, the working temperature of the filter was set to $-55^{\circ} \mathrm{C} \sim 85^{\circ} \mathrm{C}$. The separate points in Table 2 were fitted to obtain the thermal expansion curve in the temperature range. Then, the thermal expansion coefficients in the range were integrated to acquire the numerical value of each length at the corresponding varied more violently than the plate thickness with the temperature. Since $a_{l}$ is about half of $a_{2}$, the electrode size varied more violently than the plate thickness with the same temperature change. Based on these values, $\hat{\omega}_{\infty}$ and $\bar{\omega}_{\infty}$ were calculated by Eq(14). In each case, the two trapped modes of interest were found in the frequency interval.

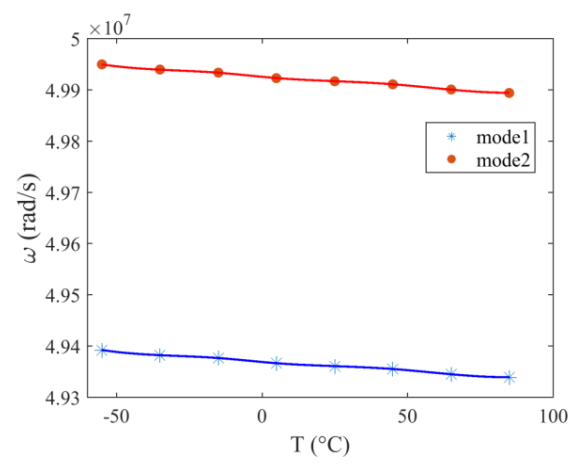

Figure 3.The frequency of trapped modes varies with temperature.

Figure 3 presents two frequency-temperature curves. The first and second modes are respectively expressed with the star line and the point line. In the first mode, the filter could operate with the in-phase vibration of the whole plate; In the second mode, the filter could also operate with the out-of-phase vibration of the dot and ring electrodes. It is clear that the temperature is negatively correlated with resonance frequency; both plate thickness and electrode size increased with the temperature. Thus, there is a negative correlation between plate thickness/electrode size andresonance frequency.

As shown in Figure 3, the frequencies varied little with temperatures. In mode 1 , the maximum and minimum frequencies were $49.3926 \times 10^{6} \mathrm{rad} / \mathrm{s}$ and $49.3395 \times 10^{6} \mathrm{rad} / \mathrm{s}$, respectively. This means the quartz crystal filter has good thermal stability.

Figure 4 show the effects of temperature on the operation modes. All parameters are the same as those in Figure 2, except that plate thickness and electrode size are variants. The figure reveals no obvious impact of temperature $(T)$ on mode shapes. This is particularly true for mode one: the three temperature curves at $-55{ }^{\circ} \mathrm{C}, 25{ }^{\circ} \mathrm{C}$ and $85{ }^{\circ} \mathrm{C}$ almost coincided completely. For mode two, there was a significant difference between the vibration modes in the ring electrode region.

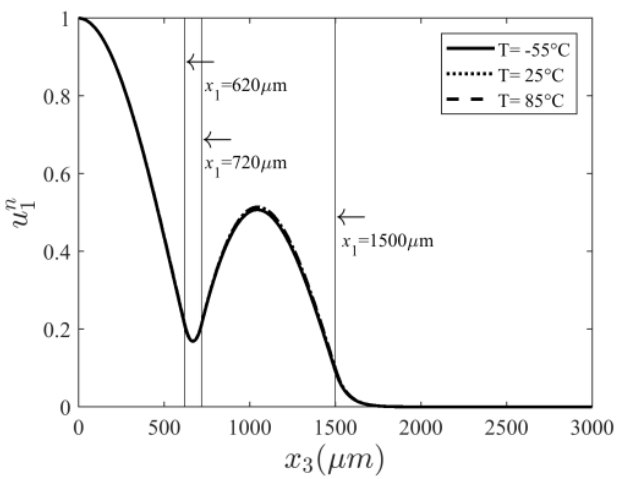

(a)

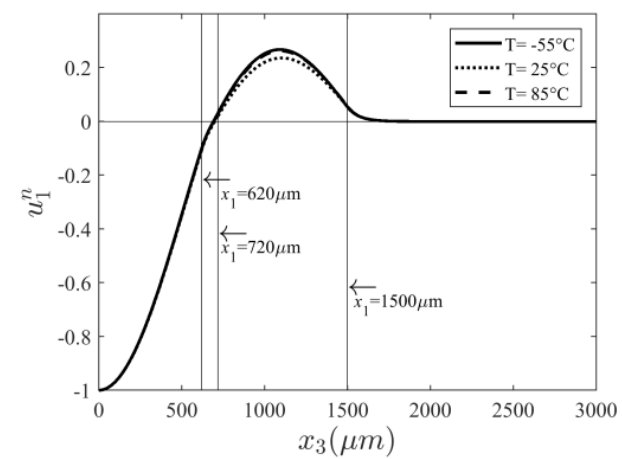

(b)

Figure 4. Effects of the temperatures $T$ on the operating modes 
The electrode scale is crucial to filter design. To disclose the effect of electrode scale, three kinds of electrode scales were designed and compared with each other. The shapes of the trapped modes are recorded in Figure 5. In the remainder of this paper, the value of $R_{2}$ is fixed as $R_{2}=1,500 \mu \mathrm{m}$, while the values of other parameters are specified in individual figures. As shown in Figure 5, the radii R0 and R1 were time-varying, resulting in changes to the area of the dot or ring electrode region. With the increase of $\mathrm{R} 0$, the vibration under the ring electrode grew weaker but remained basically the same under the dot electrode. This is because the electrode scale is positively correlated with the vibration amplitude. As R0 grew longer, the annular region shrank in size and became comparable to the central region. Thus, the vibration under the annular region attenuated. With the increase of $\mathrm{R} 1$, the vibration became weaker under the ring electrode, the annular region got smaller, the distance grew wider. A possible reason lies in the weak interaction between the two electrodes when the distance was wide.
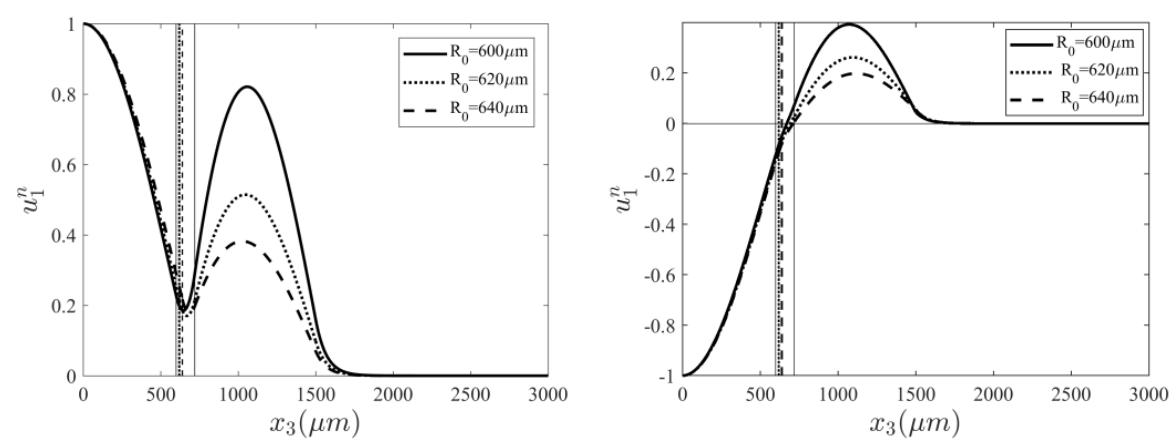

(a)
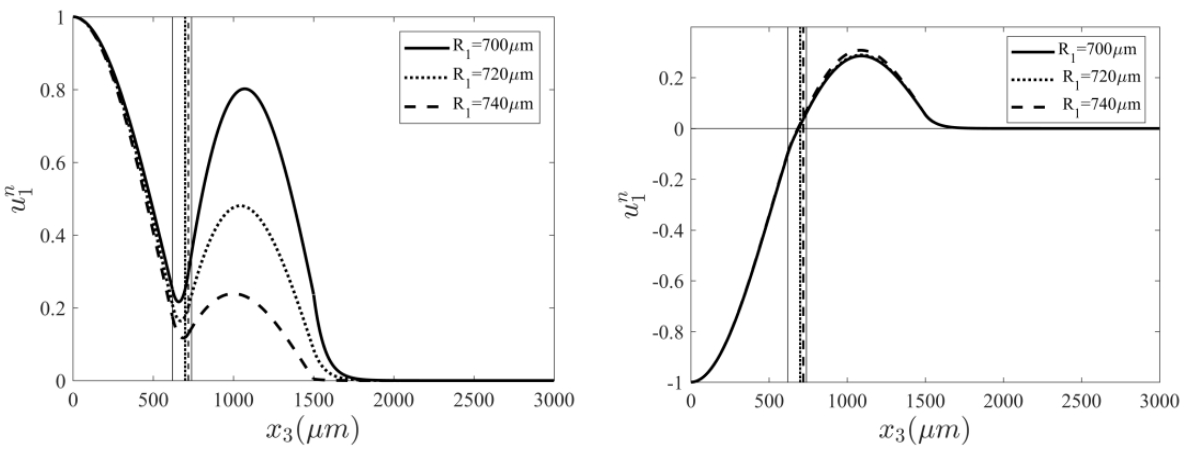

(b)

Figure 5. Effects of the radius of the electrode $R_{0}$ and $R_{1}$ on the operating modes.
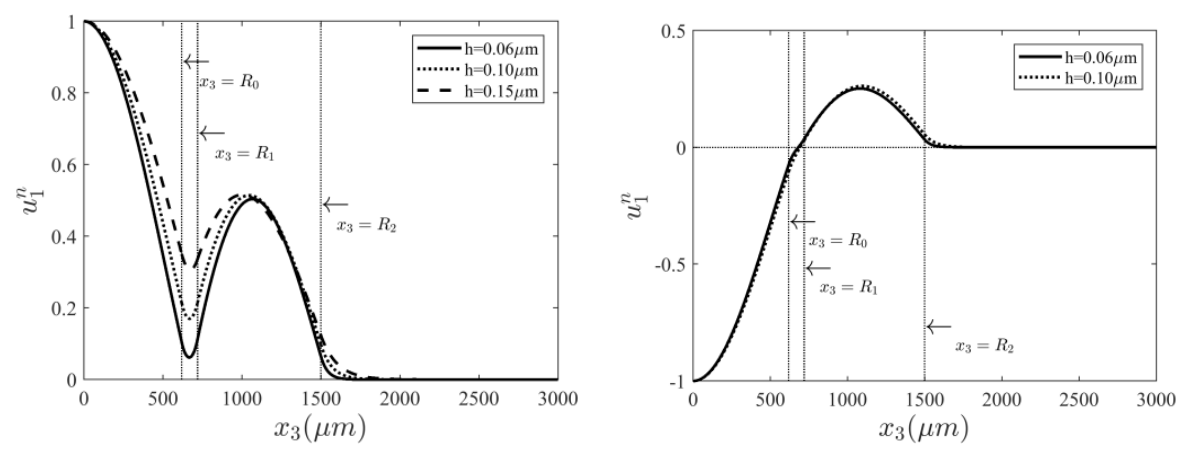

(a)
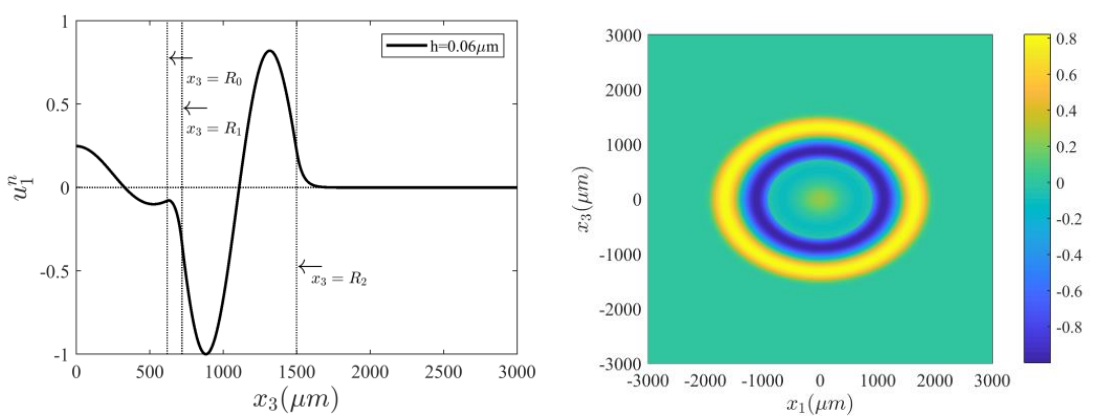

(b)

Figure 6. Effects of the half quartz plate thickness $h$ on the operating modes 
Table 3. Average and difference of the frequencies of two operating modes at different temperatures.

\begin{tabular}{ccccc}
\hline$T\left({ }^{\circ} \mathrm{C}\right)$ & $\omega_{1}(\mathrm{rad} / \mathrm{s})$ & $\omega_{2}(\mathrm{rad} / \mathrm{s})$ & $\left(f_{1}+f_{2}\right) / 2(\mathrm{MHz})$ & $f_{2}-f_{1}(\mathrm{kHz})$ \\
\hline-55 & $49,392,632.14$ & $49,949,972.75$ & $7.905,4$ & $88.703,5$ \\
-35 & $49,382,480.51$ & $49,939,629.50$ & $7.903,8$ & $88.673,0$ \\
-15 & $49,376,940.6$ & $49,933,658.95$ & $7.902,9$ & $88.604,5$ \\
5 & $49,366,806.56$ & $49,923,271.81$ & $7.901,3$ & $88.564,2$ \\
25 & $49,361,115.00$ & $49,917,211.46$ & $7.900,3$ & $88.505,5$ \\
45 & $49,355,475.27$ & $49,911,129.47$ & $7.899,4$ & $88.435,1$ \\
65 & $49,345,284.49$ & $49,900,661.59$ & $7.897,7$ & $88.391,0$ \\
85 & $49,339,532.84$ & $49,894,517.40$ & $7.896,8$ & $88.328,5$ \\
\hline
\end{tabular}

Table 4. Average and difference of the frequencies of two operating modes with different dimensions.

\begin{tabular}{ccccccc}
\hline$R o(\mu \mathrm{m})$ & $R_{l}(\mu \mathrm{m})$ & $h(\mu \mathrm{m})$ & $\omega_{1}(\mathrm{rad} / \mathrm{s})$ & $\omega_{2}(\mathrm{rad} / \mathrm{s})$ & $\left(f_{1}+f_{2}\right) / 2(\mathrm{MHz})$ & $f_{2}-f_{l}(\mathrm{kHz})$ \\
\hline 620 & 720 & 100 & $49,361,115.00$ & $49,917,211.46$ & $7.900,3$ & $88.505,5$ \\
600 & 720 & 100 & $49,479,635.10$ & $50,029,419.08$ & $7.918,7$ & $87.500,8$ \\
640 & 720 & 100 & $49,172,948.28$ & $49,863,881.04$ & $7.881,1$ & $109.965,3$ \\
620 & 700 & 100 & $49,164,215.46$ & $49,914,289.84$ & $7.884,4$ & $119.378,0$ \\
620 & 740 & 100 & $49,524,037.54$ & $49,933,262.10$ & $7.914,6$ & $65.1301,1$ \\
630 & 710 & 100 & $49,174,701.48$ & $49,880,317.05$ & $7.882,5$ & $112.302,2$ \\
610 & 730 & 100 & $49,512,410.12$ & $49,967,633.45$ & $7.916,4$ & $72.451,0$ \\
620 & 720 & 60 & $79,589,986.47$ & $79,691,441.38$ & $12.675,2$ & $16.147,1$ \\
620 & 720 & 150 & $34,281,745.84$ & -- & -- & -- \\
\hline
\end{tabular}

Figure 6 presents the effects of $h$ on the operation modes of the filter. All parameters are the same as those in Figure 2, except that plate thickness is a variant. It is observed that both modes and the number of frequency roots were sensitive to the plate thickness. Under $\mathrm{h}=0.06 \mathrm{~m}$, three trapped modes were discovered in the frequency interval. For better view, the contour of the third mode is also displayed in Figure 6. This mode contains two nodal lines: one in the dot electrode region and the other in the ring electrode region. Thus, the third mode cannot cancel out the charge in the electrode region and is not an ideal operation mode.

Our filter design adopts the average and difference of the first and second frequency roots, instead of the frequencies. When the frequencies of the two modes are close enough and their resonance peaks overlap to a certain degree, the two modes can be used together as a filter with twice the bandwidth. Tables 3-4 list the frequencies of the modes in Figures 2-6.

The frequency difference is related to the band pass width of the filter. As shown in Table 3, the average of the first and second frequency roots was close to $7.9 \mathrm{MHz}$; the frequency difference amounts to hundreds of $\mathrm{Hz}$. As shown in Table 4, the frequency difference amounts to tens of thousands of $\mathrm{Hz}$, much larger than the frequency difference in Table 3. Therefore, the thermal expansion effect on the frequency difference can be neglected in filter operation, and the accuracy of frequency should reach $\mathrm{kHz}$.

\section{CONCLUSIONS}

This paper proposes a novel "dot-ring" electrode structure for quartz filter. The corner-less design of the structure helps eliminate the concentration of electric field. Then, a theoretical analysis was performed to acquire the basic vibration features of the quartz filter with the proposed electrodes, and investigate the thermal expansion effect.

The simulation confirms the importance of the electrode size and quartz plate thickness in the design of filters. The proper design can reveal some trapped modes, whose vibrations are covered by the dot-ring electrodes. The trapped modes are the ideal operation mode, with a nodal line in the gap between the ring and the dot electrodes. Both the location of the nodal line and the field attenuation rate are sensitive to the geometric and physical parameters of the electrodes and plate. Under the working temperature between $-55{ }^{\circ} \mathrm{C}$ and $85^{\circ} \mathrm{C}$, the electrode sizes changed with the thermal expansion; the resonance frequency declined with the increase of temperature. However, the frequency difference considering the thermal expansion effect is extremely small, indicating that the quartz crystal filter has good thermal stability.

\section{ACKNOWLEDGMENT}

This research was supported by the Ningbo Natural Science Foundation in Zhejiang province, China under Grant No. 2017A610105, and the Public Projects of Zhejiang Province (No. 2015C31150).

\section{REFERENCES}

[1] Bottom VE. (1982). Introduction to quartz crystal unit design. Van Nostrand Reinhold, New York.

[2] Salt D. (1987). Quartz crystal devices, van nostrand reinhold. Wokingham, Berkshire, England.

[3] Tiersten HF. (1975). Analysis of intermodulation in thickness-shear and trapped energy resonators. Journal of the Acoustical Society of America 57(3): 667-681. https://doi.org/10.1121/1.380491

[4] Tiersten HF. (1976). Analysis of trapped-energy resonators operating in overtones of coupled thicknessshear and thickness-twist. Journal of the Acoustical $\begin{array}{llll}\text { Society of } & \text { America } & \text { 59(4): }\end{array}$ https://doi.org/10.1121/1.380947

[5] Tiersten HF, Smythe RC. (1979). An analysis of contoured crystal resonators operating in overtones of coupled thickness shear and thickness twist. Journal of the Acoustical Society of America 65(6): 1455-1460. http://dx.doi.org/10.1121/1.382907 
[6] Tiersten HF, Smythe RC. (1985). Coupled thicknessshear and thickness-twist vibrations of unelectroded ATcut quartz plates. Journal of the Acoustical Society of America $\quad 78(5)$ : 1684-1689. http://dx.doi.org/10.1121/1.392754

[7] Stevens DS, Tiersten HF. (1986). An analysis of doubly rotated quartz resonators utilizing essentially thickness modes with transverse variation. Journal of the Acoustical Society of America 79(6): 1811-1826. http://dx.doi.org/10.1121/1.393190

[8] Chen H, Wang J, Du J, Yang J. (2015). Thickness-shear modes and energy trapping in a rectangular piezoelectric quartz resonator with partial electrodes. Ferroelectrics Letters $\quad 42(1-3)$ : 10-17. http://dx.doi.org/10.1080/07315171.2015.1007801

[9] Shi J, Fan C, Zhao M, Yang J. (2015). Variational analysis of thickness-shear vibrations of a quartz piezoelectric plate with two pairs of electrodes as an acoustic wave filter. International Journal of Applied Electromagnetics and Mechanics 47(4): 951-961. http://dx.doi.org/10.3233/JAE-140090

[10] Zhao Z, Qian Z, Wang B. (2016). Energy trapping of thickness-extensional modes in thin film bulk acoustic wave filters. AIP Advance 6(1): 015002(1-11). http://dx.doi.org/10.1063/1.4939695

[11] Liu J, Du J, Wang J, Yang J. (2017). Long thicknessextensional waves in thin film bulk acoustic wave filters affected by interdigital electrodes. Ultrasonics 75: 226232. http://dx.doi.org/10.1016/j.ultras.2016.12.004

[12] Li XY, Liu J, Zhang N. (2016). Magnetoelectric coupling by acoustic wave guide. Journal of Applied Physics 119: 441-282.

[13] Li XY, Liu J, Zhang W. (2017). Modeling and characterization of mechanically mediated structure of Terfenol-D, $\mathrm{Pb}(\mathrm{Zr}, \mathrm{Ti}) \mathrm{O} 3$ and nonmagnetic Flakes. Chemical Engineering Transactions 62: 901-906. http://dx.doi.org/10.3303/CET1762151

[14] Nithyadevi N, Begum S, Shankar CU. (2015). Buoyancy and thermocapillary driven flows in an open cavity with bottom heating and symmetrical cooling from sides. International Journal of Heat and Technology 33(1): 6370. http://dx.doi.org/10.18280/ijht.330109

[15] Shockley W, Curran DR, Koneval DJ. (1967). Trappedenergy modes in quartz filter crystals. Journal of the Acoustical Society of America 41(4B): 981-993. http://dx.doi.org/10.1121/1.1910453

[16] Beaver WD. (1968). Analysis of elastically coupled piezoelectric resonators. Journal of the Acoustical Society of America 43(5): 972-981. http://dx.doi.org/10.1121/1.1910967

[17] Tiersten HF. (1969). Linear piezoelectric plate vibrations, New York: Plenum.

[18] Tiersten HF. (1977). Analysis of overtone modes in monolithic crystal filters. Journal of the Acoustical Society of America 62(6): 1424-1430. http://dx.doi.org/10.1121/1.381659

[19] He HJ, Liu JX, Yang JS. (2011). Analysis of a monolithic crystal plate acoustic wave filter. Ultrasonics 51(8): 991-996. http://dx.doi.org/10.1016/j.ultras.2011.05.015
[20] Liu J, Ma T, Du J, Wang J, Yang J. (2017). An analysis of an unbounded thin film bulk acoustic wave piezoelectric resonator with a circular driving electrode. International Journal of Applied Electromagnetics and Mechanics 56(1): 11-19. http://dx.doi.org/10.3233/JAE170014

[21] Ma Z, Duan L, Yao S. (2015). Numerical study of natural convection heat transfer in porous media square cavity with multiple cold walls based on LBM. International Journal of Heat and Technology 33(4): 69-76. http://dx.doi.org/10.18280/ijht.330409

[22] Mindlin RD. (1968). Optimal sizes and shapes of electrodes for quartz resonators. Journal of the Acoustical Society of America 973(6): 1329-1331.

[23] Yang ZY, Yang JS. (2009). Optimal electrode shape and size of a few singly rotated quartz and langasite resonators. Ultrasonics Ferroelectrics and Frequency Control IEEE Transactions on 56(2): 237-238.

[24] Liu N, Yang J, Chen W. (2011). Effects of mass layer nonuniformity on a quartz-crystal microbalance. IEEE Sensors Journal 11(4): 934-938. http://dx.doi.org/10.1109/JSEN.2010.2064297

\section{NOMENCLATURE}

$x \quad$ cartesian coordinates, $\mathrm{m}$

$h$ height,m

$R \quad$ semi-minor axis, $\mathrm{m}$

$a$ dimensionless ratio of semi-major axis/ semi-minor axis

$L \quad$ length, $\mathrm{m}$

$T$ temperature, $\mathrm{K}$

$u$ dimensionless displacement

$n \quad$ vibration order

$M$ coefficient of equation, $N / m^{2}$

$c$ elastic coefficient, $N / \mathrm{m}^{2}$

$R^{\prime}$ dimensionless electrode-plate mass ratio

$f$ frequency, $\mathrm{Hz}$

$m$ mode order

$r$ polar coordinates, $\mathrm{m}$

$\theta$ polar coordinates, rad

\section{Greek symbols}

$\alpha \quad$ thermal expansion coefficient, $\mathrm{K}^{-1}$

$\lambda$ coordinates, $m$

$\rho$ density, kg.m ${ }^{-3}$

$\varphi \quad$ angle relative to axis $\mathrm{x}_{2}$, $\mathrm{rad}$

$\eta \quad$ wave number, $\mathrm{m}^{-1}$

$\gamma$ dimensionless ratio of elastic coefficients

$\omega$ circular frequency, $\operatorname{rad} . \mathrm{s}^{-1}$

$\beta \quad$ intermediate parameters

$\xi \quad$ intermediate parameters

\section{Subscripts}

$\infty \quad$ infinite plate 